where the heart is entirely covered by lung, as shown by absence of cardiac dulness. The ready conduction of the aortic second sound along the wall of the left ventricle has been noted by Broadbent, ${ }^{15}$ who observes that it is heard at and to the left of the apex, frequently as distinctly as in the aortic area, sometimes far more so. He recommends the apex as an area over which the pure aortic sound may be studied apart from the pulmonary sound, which, he says, is not conducted along the wall of the left ventricle, though it may be heard well to the right of the apex when the right ventricle is enlarged by dilatation and hypertrophy. It is not clear to us how the pulmonary and aortic sounds can be in this manner differentiated, except on the assumption that the aortic sound passes solely along the wall of the left ventricle, not being conducted at all to the right of the septum, and, similarly, that the pulmonary sound is confined in its conduction to the wall of the right ventricle-a somewhat arbitrary route for the sound vibrations to follow.

The modifying influence of healthy lung tissue is further shown by the effect of respiration. As the lung becomes expanded on deep inspiration the second sound becomes weak, and remains weak so long as the breath is beld at the summit of inspiration; it frequently also becomes reduplicated, as has been noticed by Walshe ${ }^{16}$ and Broadbent. ${ }^{17}$ Duxing quiet breathing there is a similar, but usually less marked, variation in the intensity of the second sound, though in a few cases we have noticed the respiratory variation to be very great, even with perfectly normal respiration. With deep inspiration the margin of the left lung as defined by percussion moves downwards, so as to cover more of the heart; and over the whole of the increased area of resonauce the second sonnd becomes weaker, remaining as loud as before below the lower limit of resonance, where the heart is uncovered by lung. Not only thus in the same individual, nnder different conditions of respiration, do the anterior limits of the left lung vary, but in different individuals, we are convinced, there is great variation in the extent to which the heart is covered by lang, independently of pulmonary or cardiac disease. This opinion we are led to both by percussion and post-mortem examination. We find that the variation is much greater on the left sideaffecting the left lung-than it is on the right. Any variation in the degree to which the left lung covers the pulmonary vessel will obviously modify the londness of the pulmonary second sound, and the impression may readily arise that this sound is accentuated unless the absence or scantiness of lung-tissue be ascertained by careful percussion. A brief allusion must be made to the effect of emphysema, which we find in the majority of cases is to weaken the second sound on both sides equally, notwithstanding the high teasion which must be present in the pulmonary artery. The sounds may be extremely weak. In a case under our observation no heart sounds at all were audible over the cardiac area; they were faintly heard at the epigastrinm. Petraction of diseased lung and uncovering of the heart are common causes of apparent accentuation of the pulmonary second sound; the pulmonary artery may lio in such close apposition to the chest wall that the closure of the valves can be felt.

It has been our endearour to make it clear that the factors which determice the loudness of the second sound are namerous and compler, and that to unravel the possible causes of accentuation in any given case may be a task of great difficulty. This difficulty, we believe, can only be overcome by the consideration of other signs and symptoms, which by themselves are often sufficient for the formation of a correct diagnosis, and in the absence of which no trustwortby conclusion can be drawn from the study of the second sound. Rather than aid diagnosis the discovery of an accentuated second sound renders necessary a further and elaborate piens of differentiation if the cause of the accertuation is to be discovered.

$$
15 \text { Op. eit.. p. } 65 .
$$$$
16 \text { op. cit., p. } 75 .
$$$$
\text { IT Op. eit., p. } 68 .
$$

Eencests and Donations mo Hospitals.-By the will of the late Mr. John Steele, of Boroughbridge, Yorkshire, charities have benefited to the extent of $\Sigma 10,000$. The Farrosate Bath Hospital has received $£ 2000$. the Cottage lespital 1000 , and the Leeds Infirmary 82000 .- The late Mr. Thomas Nelson, of Newcastle. J.P., formerly a member of the City Council, has left $£ 1000$ to the Newcastle Royal Infirmary.

\section{A FURTHER CONTRIBUTION ON AN EPIDEMIC SKIN DISEASE. ${ }^{1}$}

\author{
BY THOMAS D. SAVILL, M.D. LOND.
}

WHeN attention was drawn to this malady in the year $1891,{ }^{2} 424$ cases were known to have occurred in six different institutions in London. It was defined by me as a contagious malady in which the main lesion is an inflammatory condition of the skin, sometimes attended by the formation of vesicles, always resulting in desquamation of the cuticle, usually accompanied by a certain amount of constitutional disturbance, though without pyrexia, and running a more or less definite course of seven or eight weeks.

It would appear from subsequent experience, first, that the constant presence of desquamation of the cuticle is a feature of great diagnostic value; and, second, that the disease may occur sporadically, without epjdemic relationship, and in a much milder form, especially when attacking the young. It would also appear that the outbreak of 1891 was an unusually severe one; that the disease is not rare, particulars having been collected of nearly 600 cases which arose in London during the three years 1891-93; and finally that, for reasons given, the name "general exfoliative epidemic dermatitis," suggested in 1891, besides being cumbersome, is not altogether appropriate. The term "epidemic skin disease" is preferable as indicating the conditions under which the malady first attracted attention.

The first section of the paper is occupied with a detailed description of epidemics which have been observed since 1891. In the Paddington Infirmary in the following year (1892) 12 fresh cases arose, and 2 were admitted which had arisen outside, all of which had the same character as the cases originally observed. This gave an attack rate of 2 per cent., as compared with 19 per cent. the year before, and only cne of the fourteen died.

In 18936 cases arose in the Paddington Infirmary, but none of them died.

In the Fulharn Union Infirmary, in 1893, 3 cases occurred all of which were fatal.

A very important outbreak also occurred in 1893 in the Bethnal-green Workhouse, under the care of Dr. John Knoz. ${ }^{3}$ As many as 86 cases arose in the course of five and a half weeks (May 4th to June 10th), giving an approximate attack rate of 10 per cent. amongst the persons exposed to contagion. Five of them died, giving a case mortality of nearly 6 per cent. The type of these cases was very much milder than that at the Paddington Infirmary in 1891. They had, for the most part, all the typical symptoms of the diseaseviz., anorezia, with sometimes vomiting and diarrhoe, and generally more or less weakness. Most of those attacked were middle-aged or elderly people, with one exception, a girl fourteen years of age. The skin affection began either with tiny papules or with erythema, having a tendency to symmetry, succeeded sometimes by serous exudation and always going on to desquamation of the cuticle. Though a large number of persons were attacked in a short time, the severity of the disease was much less pronounced. Evidence of this was afforded by the lower mortality, and the diminished tendency of the eruption to become universal, as compared with the Paddington and Marylebone cases in 1891.

Sporadic Cases.

Reference is next made to the fact that even in 1891 many sporadic cases came to light in the western districts of London, including one which was admitted into the Paddington Infirmary, whose history is narrated; and two others were admitted in 1892. Two sporadic cases are also referred to which were narrated in $1892{ }^{4}$ Another sporadic case occurred in the practice of Mr. Thoresby Jones, which was seen by me in consultation.

A boy aged ten years, had been attacked by an eruption three weeks before, starting in groups of red papules on the thighs, first on the right, then on the left, with more or less symmetry. A day or two later the skin became flaky, and

Abstract of a paper read in the Dermatological Section of the InterAbstract of a paper read in the $D$.
national Medical Congress at Rone.

national Medical Congress at Rome. Soe. of Lond., Nov. 5th, 1891 ; and British Journal of Dermatology, February and March, 1892.

4 Brit. Med. Jour., Jan. 9th, 1892. Drs. Cock and Evans. 
the eruption spread to other parts; and, finally, nearly the whole body, excepting the back, was involved. The temperature had been a trifle elevated at first, but afterwards it became normal. There were no other constitutional signs observed. There was considerable shedding of the epidermis in bran-like scales over the greater part of the body, but that of the hands and feet came off in large flakes. Careful investigation failed to find any possible source of infection. The disease ran its course in about five weeks, and towards the end a little brother had a very slight eruption of what was, in all probability, the same malady.

\section{CONDITIONS UNDER WHIOH THE DISEASE BECOMES} EPIDEMIO.

The malady had already been shown to be contagious, and from a study of nearly 600 cases I can now summarise the main conditions which $I$ believe to be necessary for the spread of the disease, as (1) a predisposition in those exposed to infection, by reason of advanced life, or, in a less degree, debilitated health ; (2) a collection of patients suffering from the disease in one place, favouring, it would seem, a concentration of the poison; and (3) certain obscure seasonal and meteorological conditions.

Nearly all the known cases have arisen between May and November-i.e., during the summer and autumn months-and there is certainly a marked tendency towards the spread of the disease at these seasons. As regards the other conditions necessary, no very precise information is yet attainable. In view of the fact that the disease in 1891 spread from ward to ward with great rapidity, the distance necessary for contagion need not apparently be very close ; but, on the other hand, the free communication between these wards by means of medical attendants and nurses-for no isolation was attempted-would negative the value of this observation. But, again, the rapidity with which the disease spread in the Bethnal-green Workhouse, where eighty-six persons were attacked in different parts of the buildings in the short space of five weeks and a half, and where isolation was in some measure adopted, would tend to show that the malady may be spread some distance through the air.

Diagnosis.

The large number of cases which have come to light during and since the year 1891 support the view that the disease is by no means a rare one. It is during the initial stage that mistakes in diagnosis are most liable to occur. At this time it may resemble many differently named diseases. The commonest mode of commencing is a papular rash, arranged more or less in constellations, and usually attacking symmetrical parts of the body. Next in order of frequency is an erythema. In mild cases this initial stage is very transient, lasting only a day or two. It is rapidly succeeded by the characteristic desquamation of the cuticle, which not only occurs in parts where the papules or ery thema have been observed, but also in other places. In severe cases the papular or erythematous eruption is succeeded by thickening of the skin, which has a red excoriated surface, throwing off it: epidermis in successive coats. Cases of average severity are without donbt more likely to be confused with eczema than with any other disease. The following are the chief clinical distinctions :-

$$
\text { Ecrema. }
$$

1. Attacks all ages, and children are very liable.

2. Gout is a marked predisposing canse.

3. Constitutional disturbance always moderate and never fatal.

4. Dried crusts thrown off, bat exfoliation of cuticle not a marked feature of the disease. Dermal thickening absent or moderate.

5. Exudation generally more or less present.

6. Course not definite.

7. Not hitherto regarded as contagious or epidemic.
Epidemic Skin Disease.

1. Cnildren almost exempt; old people specially prone.

2. Gout offers no predisposi. tion.

3. Constitutional disturbance often severe, and may be fatal.

4. Kpidermal exfoliation a very constant feature. It mav occur in some places without previons exup. tion. Dermal thickening generally present.

5. Fixudation may be slight, transient, or absent.

6. Course fairly definite.

7. Undoubtedly contagious, and epidernic ander certain conditions. commencement as a papular or erythematous eruption, which is followed in a few days by (2) desquamation of the cuticle (a symptom which is never absent) in bran-like scales or flakes; and (3) its more or less definite duration of six or eight weeks.

\section{BACTERIOLOGY.}

Both Dr. Risien Russell and I detected a characteristic diplococcus in the exudation and blood of the cases in 1891, but in two subsequent attempts I have been unsuccessfal in obtaining characteristic cultures.

TREATMENT.

The efficacy of the creolin bath is again emphasised, and cases are mentioned where patients have relapsed on its cessation and recovered again on its renewal. Two pints and a balf of a 1 per cent. solution are added to fifteen gallons of comfortably warm water, and the bath is adninistered once or twice a day. Good effects were also obtained from a $1 \mathrm{cr}$ 2 per cent. creolin ointment, ichthyol and har scap, liquor carbonis detergens ( 1 drachm to the vint), Unna's oxide of zinc gelatin, and other remedies; but corrosive sublimate solution always did harm.

\section{NOSOLOGY.}

In its clinical features I still congider the disease to belong to the eczematous rather than to any other group of skin diseases, though visible exudation may very of ten be absent. It might be said that the constant presence of exfoliation forms an objection to its being so classified, and that it belongs to the exfoliative disorders; but it may also be urged, firstly, that pityriasis rubra, the most typica of exfoliative dermatites, which the epidemic skin disease also resembles, has been regarded as an eczematous disease by some authorities ${ }^{5}$ and, secondly, that the exudation, though apparently absent, has been detected on the under surface of the scales in some cases.

[In conclusion, attention was drawn to the interestiog questions which arise out of a study of this disorder. The variety often presented by the rash, especially in its early stage, is a feature of considerable interest to those who study skin diseases from a clinical standpoint; for the essentid cause of the different appearances is probably the same in all cases, though the resulting conditions resemble many different skin affections hitherto described by different names. To the pathological student it is of equal interest, not only by reason of certain points in its etiology and epidemic occurrence, but also from the part, presamably played by microbss in the origin and development of this. curious skin affection.]

Upper Berkeley-street, $\mathrm{W}$.

\section{THE ACTION OF METHYLENE BLUE ON LÖFELER'S BACILII, WITH CLINICAL SUGGESTIONS.}

BY BERTRAM HUNT, M.B. OZON., H.R.C.S. ING。

IT is impossible to work with Iöfler's bacill without remarking the rapid and differential stain imparted to them by dilute solutions of methylene blue. Before entering upon the subject of this paper-namely, the action of the blae on the living bacilli-it seems worth while to mention a method I have found affording very pretty preparations, and to briefly describe the staining reaction generally, seeing that it led me to make my research, and forms, therefore, a fitsing introduction. If bacilli grown for twenty-four hours on blood serum or on glycerive agar be thickly smeared with the needle on to the centre of a cover-glass. and if after drying and passing through the flame a drop or two of dilutes stain be added to them, the colour is observed to enter the bacilli so quickly that in a few seconds the solution in their neighbournood becomes colourless. On removing the excess of stain with filter paper and examining the preparation under the microscope, the differential character is at once apparent. The bacilli take only a comparatively light tixt, but in some of them one or two dark blue points (pole granules) are visible, one at each end perhaps; in others, which are longer, three or four, one at each end, and one or two in the middle, marking, presumably, stages in fissioz:

The three chief means of distinguishing the skin disease under consideration from other skin affections are (1) its 